\title{
PROPERTIES OF STRONG GROUND MOTION EARTHQUAKES*
}

\author{
By George W. Housner
}

ThE usual type of California earthquake is associated with a relative horizontal slipping of the two faces of a fault which lies essentially in a vertical plane. As a consequence of this slipping, the surface of the ground experiences a severe motion in the neighborhood of the fault during large earthquakes. This surface ground motion can be measured, and for the relatively small number of strong ground motions that have been recorded the maximum horizontal acceleration measured was 0.33 of gravity. For obvious reasons the accumulation of data on strong ground motion is a slow process, so that it will be many years before a sufficiently large body of observations is amassed to give a reasonably complete picture of what can be expected in the way of destructive seismic motions. Analysis of the problem is rendered difficult by the fact that it is not possible to make direct observations of the mechanism that generates the seismic waves, for the usual California earthquake originates at a depth of approximately ten miles, although in some large earthquakes the slipping on the fault has extended to the surface of the ground.

From a certain point of view many of the observed properties of destructive shocks can be correlated to give a consistent picture of what happens in the large, although because of the complexity many of the details cannot be accounted for precisely. The slipping that takes place along an active fault releases shear stress that existed on the fault, and the rapid release of stress sends out waves which, after reflection and refraction through various strata, produce the motion that is measured on the surface of the ground. If the initial state of stress on the fault is not uniform, and if the stress on the fault during slipping is not constant, some relatively short-period stress waves may be sent out that would have a strong effect on the ground acceleration measured near the fault, even though their effect would be negligible on recorded ground displacements. The properties of strong groundmotion accelerograms will thus reflect the direction of slip, the size of the slip area, the strata through which the waves pass, and the details of nonuniformity of the stress that is released.

The slipping along a fault may be pictured as a releasing of shear dislocations. An example of a shear dislocation, as defined in this paper, is shown in figure $1, a$ and $b$. Consider an indefinitely extended elastic material, in the interior of which there is a plane crack with boundary " $a$ " as shown in figure 1 , $a$. The two abutting faces of the crack have been given a lateral displacement relative to each other as shown in figure $1, b$. In order to maintain this relative displacement there must exist equal and opposite shear stresses on the two faces. These shear stresses can exist only if friction forces can be developed and this requires that compressive stresses exist in the material. In a typical single dislocation the stress on the faces of the dislocation and in the adjacent material will be distributed in the manner shown in figure 1, $c$ and $d$. The precise form of the shear distribution will depend upon the relative displacement of the two faces and the shape of the dislocation. If the

\footnotetext{
* Manuscript received for publication February 4, 1954.
} 
stressed dislocation suddenly snaps to the unstressed position, stress waves will be propagated through the surrounding medium and thus produce an elemental earthquake.

If a cluster of overlapping dislocations were spread over a plane area they would form a large crack which may be called a composite dislocation. The shear stresses on the faces of the composite dislocation would vary over the area, depending upon

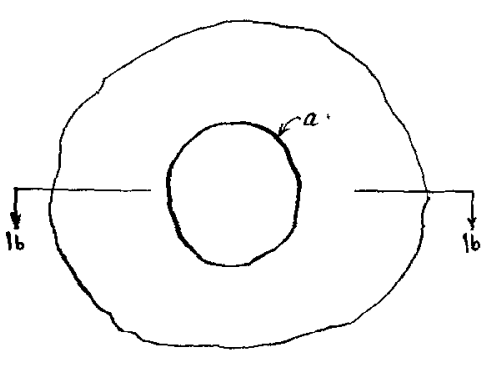

$a$

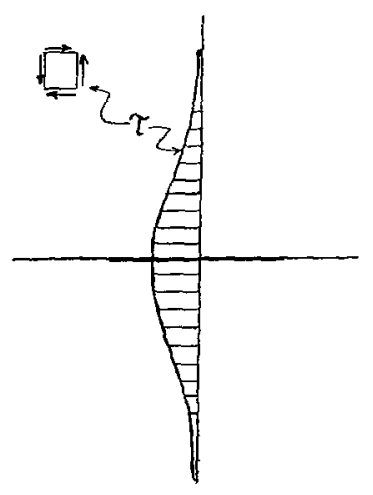

$d$
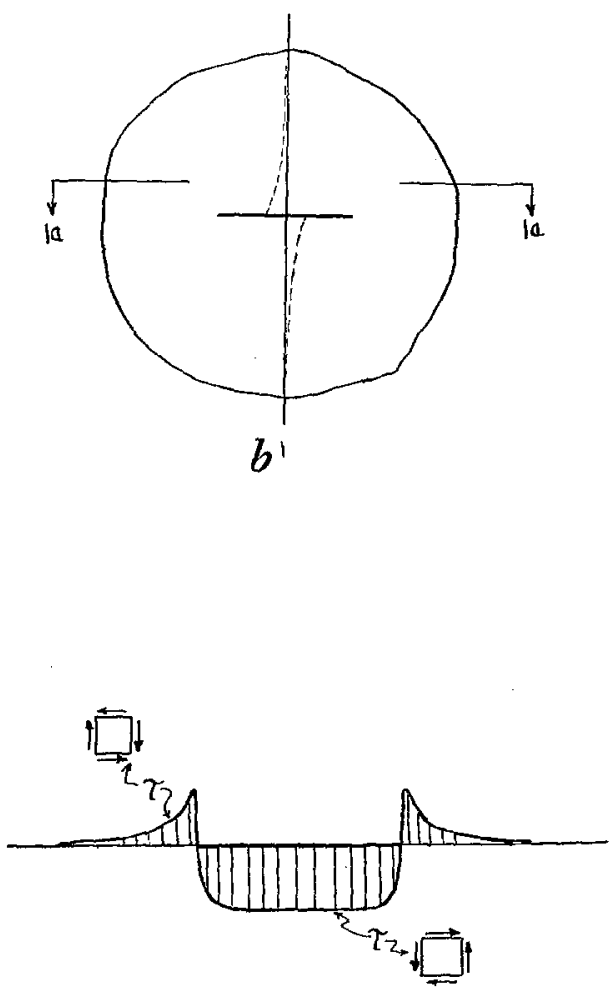

c

Fig. 1. Stress on a dislocation.

the stresses of the incremental dislocations of which it is formed and upon the manner of superposition. The stresses associated with a shear dislocation are selfequilibrating in such a manner that an earthquake fault may be considered to be formed by a planar distribution of shear dislocations; that is, these form the crack, and superposed is a state of shear produced by relative horizontal translation of separated points of the earth's crust. The superimposed shear strain is relieved by release of the dislocations. The notion of shear dislocations is merely a convenient concept for describing the release of stress. Any kind of slipping can be described by an appropriate distribution and release of shear dislocations and, in particular, the influence of nonuniformity of stress may be deduced from them. 


\section{Release of Dislocations during an Earthquake}

If an earthquake fault is considered to be formed by a distribution of a large number of dislocations with a superposed state of shear, then as the stresses are built up in the earth's crust eventually the stress at one of the dislocations will exceed the limiting stress and that dislocation will snap. If that point on the fault is surrounded by a region of relatively low stress, the slipping will stop and a small, single-dislocation earthquake will have been generated. If the region around this point has a high stress, the initial release will increase the stress on the surrounding region, and slipping will progress. If the stress originally on the fault is not uniform and the stress on the fault during slipping is not uniform, the effect of these irregularities of stress may be thought of as releases of dislocations. The ground motion produced by the release of shear stress over a localized region may be investigated as follows.

The displacements generated by the application of a concentrated force at an interior point of an extended, homogeneous, isotropic, linearly elastic solid are given by the expressions ${ }^{1}$ which follow.

$$
\begin{aligned}
& u=\frac{1}{4 \pi \rho}\left[\frac{1}{r b^{2}} F\left(k t-\frac{k r}{b}\right)+\frac{1}{r}\left(\frac{\partial r}{\partial x}\right)^{2}\left\{\frac{1}{a^{2}} F\left(k t-\frac{k r}{a}\right)-\frac{1}{b^{2}} F\left(k t-\frac{k r}{b}\right)\right\}\right. \\
& \left.+\frac{\partial^{2} r^{-1}}{\partial x^{2}} \int_{r / a}^{r / b} t^{\prime} F\left(k t-k t^{\prime}\right) d t^{\prime}\right] \\
& v=\frac{1}{4 \pi \rho}\left[\frac{1}{r} \frac{\partial r}{\partial x} \frac{\partial r}{\partial y}\left\{\frac{1}{a^{2}} F\left(k t-\frac{k r}{a}\right)-\frac{1}{b^{2}} F\left(k t-\frac{k r}{b}\right)\right\}\right. \\
& \left.+\frac{\partial^{2} r^{-1}}{\partial x \partial y} \int_{r / a}^{r / b} t^{\prime} F\left(k t-k t^{\prime}\right) d t^{\prime}\right] \\
& w=\frac{1}{4 \pi \rho}\left[\frac{1}{r} \frac{\partial r}{\partial x} \frac{\partial r}{\partial z}\left\{\frac{1}{a^{2}} F\left(k t-\frac{k r}{a}\right)-\frac{1}{b^{2}} F\left(k t-\frac{k r}{b}\right)\right\}\right. \\
& \left.+\frac{\partial^{2} r^{-1}}{\partial x \partial z} \int_{r / a}^{r / b} t^{\prime} F\left(k t-k t^{\prime}\right) d t^{\prime}\right]
\end{aligned}
$$

where $u, v, w$ are the displacements in the $x, y, z$ directions and the force is applied at the origin in the $x$ direction, and

$$
\begin{aligned}
F(k t) & =\text { magnitude of force as function of time. } \\
t & =\text { time } \\
k & =\text { constant } \\
r & =\left(x^{2}+y^{2}+z^{2}\right)^{1 / 2} \\
a & =\text { velocity of dilatational waves } \\
b & =\text { velocity of rotational waves } \\
\rho & =\text { density }
\end{aligned}
$$

${ }^{1}$ A. E. H. Love, The Mathematical Theory of Elasticity (Cambridge University Press, 1934), 
It will be noted from the preceding expressions that if the force is initially applied at time $t=0$, a point at a distance $r$ from the origin will remain undisturbed until time $t=r / a$, when the dilatational waves reach it. At time $t=r / b$ the rotational waves reach the point.

If we think of the $z$ axis as being vertical and the preceding expressions are differentiated with respect to $y$, the resulting equations describe the motion generated by a pair of equal and oppositely directed forces one of which is applied at the origin in the negative $x$ direction and the other is applied at the point $x=0, y=\epsilon, z=0$ in the positive $x$ direction, $\epsilon$ being an infinitesimal. This may be interpreted as an infinitesimal dislocation of area $(d x d z)$ with shear stresses $\tau_{x z}= \pm F(k t) / d x d z$ applied to the faces. The displacements are given by

$$
\begin{aligned}
(4 \pi \rho) u=- & \frac{k}{b^{3}} \frac{y}{r^{2}}\left(1-\frac{x^{2}}{r^{2}}\right) F^{\prime}\left(k t-\frac{k r}{b}\right)-\frac{k x^{2} y}{a^{3} r^{4}} F^{\prime}\left(k t-\frac{k r}{a}\right)-\frac{y}{b^{2} r^{3}}\left(1-\frac{x^{2}}{r^{2}}\right) F\left(k t-\frac{k r}{b}\right) \\
& -\left\{\frac{y x^{2}}{a^{2} r^{5}} F\left(k t-\frac{k r}{a}\right)-\frac{\partial^{3} r^{-1}}{\partial x^{2} \partial_{y}} \int_{r / a}^{r / b} t^{\prime} F\left(k t-k t^{\prime}\right) d t^{\prime}\right\} \\
(4 \pi \rho) v=\frac{k}{b^{3}} & \frac{x y^{2}}{r^{4}} F^{\prime}\left(k t-\frac{k r}{b}\right)-\frac{k}{a^{3}} \frac{x y^{2}}{r^{4}} F^{\prime}\left(k t-\frac{k r}{a}\right)+\left\{\left(\frac{x}{r^{3}}-\frac{3 x y^{2}}{r^{5}}\right)\right. \\
& \left.\cdot\left(\frac{1}{a^{2}} F\left(k t-\frac{k r}{a}\right)-\frac{1}{b^{2}} F\left(k t-\frac{k r}{b}\right)\right)+\frac{\partial^{3} r^{-1}}{\partial x \partial y^{2}} \int_{r / a}^{r / b} t^{\prime} F\left(k t-k t^{\prime}\right) d t^{\prime}\right\} \\
(4 \pi \rho) w= & \frac{k}{b^{3}} \frac{x y z}{r^{4}} F^{\prime}\left(k t-\frac{k r}{b}\right)-\frac{k}{a^{3}} \frac{x y z}{r^{4}} F^{\prime}\left(k t-\frac{k r}{a}\left(+\left\{\frac { 3 x y z } { r ^ { 5 } } \left(\frac{1}{a^{2}} F\left(k t-\frac{k r}{a}\right)\right.\right.\right.\right. \\
& \left.\left.-\frac{1}{b^{2}} F\left(k t-\frac{k r}{b}\right)\right)+\frac{\partial^{3} r^{-1}}{\partial x \partial y \partial z} \int_{r / a}^{r / b} t^{\prime} F\left(k t-k t^{\prime}\right) d t^{\prime}\right\}
\end{aligned}
$$

where $F^{\prime}$ is the derivative of $F$.

The appropriate integrations over an area $A$ in the $x-z$ plane will then give the disturbances generated by the application of horizontal shear stresses to the forces of a dislocation of area $A$ lying in the $x, z$ plane. At a point the distance of which from the origin is large compared to the dimensions of $A$, the displacements are essentially given by the preceding equations when $F(k t)$ is interpreted as the resultant horizontal shear force on the face of the dislocation. If the force is applied rapidly ( $k$ being large), the last term in each of the expressions given above will be small compared to the other terms. The dilatational and rotational components of the terms are similar; hence the characteristics of the motion can be studied by considering the rotational waves only. These produce the motion

$$
\begin{aligned}
& u=-\frac{k}{4 \pi \rho b^{3}} \frac{y}{r^{2}}\left(1-\frac{x^{2}}{r^{2}}\right) F^{\prime}\left(k t-\frac{k r}{b}\right) \\
& v=\frac{k}{4 \pi \rho b^{3}} \frac{x y^{2}}{r^{4}} F^{\prime}\left(k t-\frac{k r}{b}\right)
\end{aligned}
$$




$$
w=\frac{k}{4 \pi \rho b^{3}} \frac{x y z}{r^{4}} F^{\prime}\left(k t-\frac{k r}{b}\right)
$$

These expressions show that the ground displacements are directly proportional to the time rate of change of the applied forces. Consider a circular dislocation with an initial static shear stress $s_{1}$ on the faces, and assume that slipping starts at the center of the dislocation and progresses with uniform velocity to the perimeter. During slipping the stress on the face of the dislocation will drop to a value $s_{2}$
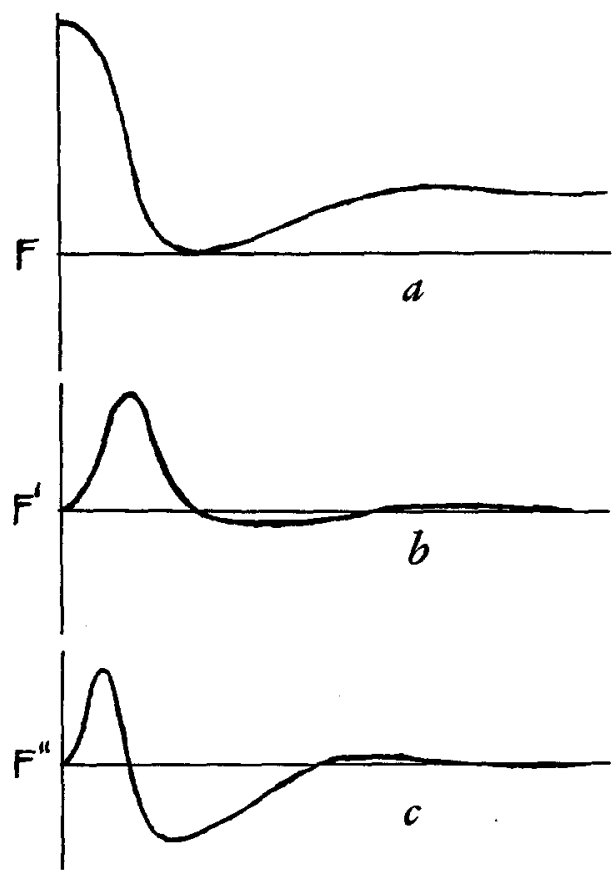

Fig. 2. Force on a dislocation as a function of time. $a$, resultant force on face; $b$, displacement of point with passage of wave; $c$, velocity; $d$, acceleration.

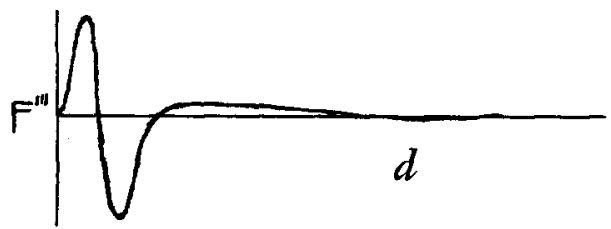

depending on sliding friction. The relative motion between the faces of the dislocation is brought to rest progressively from the perimeter to the center of the dislocation. During this process the stress will drop to essentially zero. Following this the stress will build up to some value $s_{3}$ as the surrounding material comes to rest: The resultant force on the face of the dislocation will vary with time essentially as shown in figure 2, a, with successive derivatives as shown in figure $2, b, c, d$. The displacement at a point, produced by the passage of the waves, will vary as shown in figure $2, b$, the velocity as shown in figure 2, $c$, and the acceleration as shown in figure $2, d$. It is seen that the rotational wave generates essentially a single displacement pulse and a double acceleration pulse, this latter resembling one cycle of a sine wave. The dilatational wave produces similar pulses traveling at a higher velocity. 
The preceding expressions also show that points on the $x, z$ plane do not undergo any motion. This indicates that if the earth's crust were homogeneous and isotropic there would be no motion on the fault plane outside the area of slip. Nonhomogeneities, and the like, in the earth's crust produce wave reflections and refractions, so that motion does occur on the fault plane at the surface of the ground; but some observations have shown that the intensity of ground motion near the fault plane is less than at adjacent points.

It may also be noted from the preceding expressions that there is a strong directional effect in that the amplitude of motion is very much less in the vicinity of the $x$ axis than in the vicinity of the $y$ axis. This indicates that for vertical slipping on a fault the epicentral region will be shaken with very much less intensity than if the same energy were released by horizontal slipping. It also indicates that more energy would be radiated parallel to the fault for vertical slipping than for horizontal slipping. Both of these effects were reported on the occasion of the Arvin-Tehachapi earthquake of July, 1952, which was stated to have been produced by essentially vertical slipping.

\section{The Mechanism of Release}

To determine when and where the individual dislocations are released during an earthquake would require a precise knowledge of the state of stress along the fault, the properties of the material, and other facts. This is clearly impossible to achieve; however, the problem may be approached statistically. That is, a probability mechanism can be postulated that, although not describing precisely the release of each individual dislocation, will describe the average properties of ensembles of dislocations. Again, there is no way of deriving a precise specification of the statistical problem, but this must be deduced from observations of actual earthquakes, a knowledge of the properties of rock, a knowledge of stress distributions associated with shear dislocations of specified type, and the like. From such considerations the following mechanism is postulated.

First, the average relative slip between the faces of a composite dislocation of area $A$ is taken to be proportional to the square root of $A$. This statement is to be understood in a probability sense. That is, it is not required that for every composite dislocation the average slip be precisely proportional to the square root of the area of slip; it is only required that the deviations from this rule may be viewed as statistical deviations about a mean and that the statements refer to the relation between the mean slip of all earthquakes of a specified area and that area.

The statement that the average slip is proportional to the square root of the slip area implies that the areas are geometrically similar. Actually this can be true only for slip areas up to a certain size, for the usual strong-motion California earthquakes originate at a ten-mile depth and the extent of the slip area is bounded by the surface of the ground. The growth of the slip area is also inhibited because at increasing depths the physical properties of the rock approach those of a material which flows plastically under applied stresses and therefore cannot maintain shear stresses of as great magnitude as the material nearer to the surface, although it undoubtedly can rupture in a brittle manner under suddenly applied shear strains. It is presumed that slip areas of increasing size will be geometrically similar, roughly circular in shape, up to a certain size $A_{1}$; from size $A_{1}$ to $A_{2}$ the shapes of the slip areas are not 
geometrically similar; for areas greater than $A_{2}$ geometrical similarity again holds, the shapes of the areas being roughly rectangular and differing in length of slip but not appreciably in depth of slip area. For this last case the average slip is proportional to the length rather than the square root of the area.

Second, there is required a formulation of the probability of release of dislocations. The mechanism that controls the size of the slip area is associated with the distribution of static shear stress along the fault. It is logical to assume that the distribution of shear stress varies quite irregularly over the fault plane, that is, something like a two-dimensional, random, continuous function. As the state of shear strain builds up, the stresses increase until some point reaches the failing stress. This region will then slip and the extent of the slip will be governed by the region of low stress surrounding this point. The state of stress over a rectangular area of fault with dimensions $l_{1}$ by $l_{2}$ can be expressed by

$$
\tau=\sum \sum C_{m n} \sin \frac{n \pi}{l_{1}} x \sin \frac{m \pi}{l_{2}} y
$$

and as the state of strain builds up, the coefficients $C_{m n}$ increase until a slip occurs, at which time there is a sudden change in the coefficients. The relative displacement of the two faces of the fault from the unstressed configuration will also be irregular, but will be much smoother than the stress distribution. The relative displacement can also be expressed in a Fourier form:

$$
u=\sum \sum B_{m n} \sin \frac{n \pi}{l_{2}} x \sin \frac{n \pi}{l_{1}} y
$$

It is assumed that the $B_{m n}$ occur in such a way that the frequency of slips of various areas is inversely proportional to the area. That is, the expected number of slips having areas lying between $A$ and $A+d A$ is proportional to $d A / A$. As will be seen, such a frequency distribution for the areas agrees well with observations. It is possible, in principle, to compute the detailed behavior of the coefficients $C_{m n}$ and $B_{m n}$ required for such a frequency distribution, and this would give information on the probable distribution of stress and strain along a fault.

Let the frequency distribution of $\operatorname{slip}$ areas $A$ be written

$$
f=C \frac{a_{0}}{A}=C \frac{1}{x}
$$

where $a_{0}$ is the lower limit of $A$. The total probability must equal unity, so that

$$
\begin{gathered}
\int_{x_{0}}^{x_{2}} \frac{C}{x} d x=C \log \frac{x_{1}}{x_{0}}=1 \\
C=\frac{1}{\log \frac{x_{1}}{x_{0}}}
\end{gathered}
$$


where $x_{1}$ is the maximum possible value of $x$. The frequency distribution of $x$ is therefore

$$
f=\frac{1}{\log \frac{x_{1}}{x_{0}}} \frac{1}{x}
$$

It may be noted that there must be both an upper limit and a lower limit for $x$, otherwise $\log x_{1} / x_{0}$ becomes infinite and the frequency distribution does not exist.

The mechanism postulated above does not describe the details of a specific earthquake, but it describes an average process that should agree with the averages of observations. The mechanism is also a simplification in that it does not include the effects of the variation of physical parameters in the earth's crust. In particular, the fact that with increasing depth the physical properties vary from those of an essentially linearly elastic solid to those of a material that deforms plastically under slowly applied strains is not included. This means that certain relaxation processes that are undoubtedly operative are not considered. Also, the mechanism excludes from consideration such shocks as may arise from processes other than horizontal slipping along essentially vertical fault planes.

\section{Magnitude and Frequency}

According to the view expressed above, a natural measure of an earthquake is the average slip occurring on the fault. Accordingly, a logarithmic measure of the average slip is taken to define an earthquake, that is, the measure $M$ is related to the average slip by

$$
d M=\frac{d \bar{S}}{S}
$$

where $\bar{S}$ is the average relative slip. In integral form this is

$$
\bar{S}=\bar{S}_{0} e^{M}
$$

Since the average slip is proportional to the square root of the slip area $A$ it follows that

$$
A=A_{0} e^{2 M}
$$

The frequency distribution of earthquakes given by equation (1) states that the relative frequencies are inversely proportional to the area $A$, so the frequency distribution of shocks in terms of $M$ is

$$
f=c e^{-2 M}
$$

Equation (3) may be compared with recorded data on frequency of occurrence of earthquakes. Such data have been presented by Gutenberg and Richter, ${ }^{2}$ who give

\footnotetext{
${ }^{2}$ B. Gutenberg and C. F. Richter, Seismicity of the Earth (Princeton University Press, 1949).
} 
the observed frequencies of shallow-focus world earthquakes, southern California earthquakes, and New Zealand earthquakes as

$$
\log _{10} N=a+b(8+M)
$$

where $N$ is the mean annual number per tenth magnitude, $M$ is the magnitude of a shock as defined by Richter, and the coefficient $b$ is $0.9,0.88,0.87$, respectively. These values were obtained by plotting $\log N$ against $M$ and fitting a straight line to the data. The points fitted the line closely except for magnitudes greater than 8 , in which range the observed frequencies fell below the line with an apparent upper limit for $M$ of 8.7. If the preceding equation is put in exponential form, there is obtained for world earthquakes, southern California earthquakes, and New Zealand earthquakes, respectively,

$$
\begin{aligned}
& N=c_{1} e^{-2.07 M} \\
& N=c_{2} e^{-2.02 M} \\
& N=c_{3} e^{-2.00 M}
\end{aligned}
$$

It is seen that equation (3) fits the data within the limits of observational accuracy.

It is also seen from the foregoing that the measure of an earthquake as defined in this paper may be identified with the magnitude defined by Richter. He defined the magnitude as being the logarithm of the maximum trace amplitude recorded by a certain type of seismograph located 100 kilometers from the epicenter of an earthquake. It will be shown later that the maximum acceleration is proportional to the square root of the area of slip, and hence that the physical interpretation of the two measures is the same.

\section{Energy Released}

The strain energy stored in a solid by a single shear dislocation is equal to the work that must be expended by the shear stresses acting on the faces of the dislocation in producing the relative displacement of the faces. The strain energy stored by a dislocation of area $a_{n}$ is, thus,

$$
E_{n}=\frac{1}{2} \int \tau_{n} S_{n} d a_{n}
$$

where $\tau_{n}$ is the shear stress acting on the face and $S_{n}$ is the relative displacement (variable) of the two faces. The failing stress $\tau$ is the same for all dislocations, so if $\bar{S}_{n}$ and $\bar{a}_{n}$ are the average slip and average area the total energy released by $N$ dislocations is

$$
E=\sum_{n=1}^{N} E_{n}=\sum C_{n} \tau \bar{S}_{n} \bar{a}_{n}=\bar{C} N
$$

The total energy released is thus proportional to the number $N$ of dislocations released. The number $N$ is proportional to the area of slip multiplied by the average slip over $A$, so 
or

$$
E=C_{1} A \bar{S}
$$

$$
E=C e^{3 M}
$$

This relates the energy released to the magnitude of the shock.

Equation (4) applies only to the smaller geometrically similar slip areas. For example, a small slip area will have longitudinal and vertical dimensions approximately equal, and increasingly larger slip areas will be geometrically similar to this up to a certain point. The vertical dimensions of slip areas are limited in that a slip originating at a ten-mile depth can extend upward only ten miles, and because of the changing physical properties of the earth's crust with depth, the slip is limited in the downward direction also. This means that the slip areas of very large earthquakes increase principally by an elongation of the area and that the vertical dimension of the slip area does not increase proportionally. Such slip areas are not geometrically similar to the smaller slip areas, and this must be taken into account when the energy released is computed. For geometrically similar slip areas the average slip is proportional to the length of the fault. For the smaller shocks this varies as

$$
\bar{S}=C^{\prime} \sqrt{A}
$$

However, for very large geometrically similar shocks where the slip area receives no restraint from material above or below, the length is proportional to the area, so that

$$
\bar{S}=C^{\prime \prime} A
$$

In this case the total energy must be written

$$
E=C e^{4 M}
$$

There is, of course, a transition region between the limits of applicability of equations (4) and (4a), and the latter presumably applies only to very large shocks.

The foregoing expressions for energy released may be compared with those derived by an alternate method by Gutenberg and Richter. ${ }^{3}$ Consider that at the epicenter the radiated energy arrives in a sinusoidal wave train with maximum acceleration $a_{0}$ and duration $t_{0}$, that is, consider the actual accelerogram to be replaced by an equivalent sinusoidal wave train. Assuming the total energy released to be proportional to the energy reaching the epicenter will then give

$$
E=C t_{0} a_{0}^{2}
$$

where $a_{0}$ is the maximum acceleration at the epicenter. From the recorded ground accelerations it is found that the following empirical expression relates $a_{0}$ with $M$

$$
M=2.2+1.8 \log _{10} a_{0}
$$

or

$$
a_{0}=C_{1} e^{1.28 M}
$$

${ }^{3}$ B. Gutenberg and C. F. Richter, "Earthquake Magnitude, Energy, Intensity and Acceleration," Bull. Seism. Soc. Am., 32: 163-191 (1942). 
As shown below, the duration may be taken to be

$$
t_{0}=C_{2} e^{M / 2}
$$

These values give for the energy released

which agrees with equation (4).

$$
E=C e^{3.06 M}
$$

The foregoing analysis does not include the energy carried in the long-period components that are found in very large shocks. To allow for this, Gutenberg and Richter apply the correction factor $e^{M}$, thus obtaining

$$
E=C e^{4.06 M}
$$

which agrees with equation (4a).

\section{Areas of Slip}

According to the foregoing analysis, the total area of relative slip along a fault is proportional to the square of the maximum slip. In terms of the magnitude the area of slip is thus

$$
A=A_{0} e^{2 M}
$$

This expression should be understood in a probability sense, namely, that on the average shocks of magnitude $M$ will have an area $A$ corresponding to equation (5). As was seen when discussing equation (1), there must be an upper limit for the area $A$ and also a lower limit. The lower limit is the area of the smallest individual shear dislocation that can be released under the conditions applying to an earthquake fault; the upper limit is imposed by the fact that earthquake faults are of finite extent.

The areas corresponding to different values of $M$ can be compared by means of equation (5), for example,

$$
\frac{A_{1}}{A_{2}}=e^{2\left(M_{1}-M_{2}\right)}
$$

To investigate the implications of this equation let the El Centro shock of May 18, 1940, be considered a typical 6.7 magnitude earthquake. Judging from the visible surface slip, it is estimated that this shock had a slip area of approximately $40 \times 20$ $=800$ square miles. Using this as a base, the areas of shocks of other magnitudes are given by

$$
A=800 e^{2(M-6.7)}
$$

This gives for a shock of magnitude $M=0$, an area of slip

$$
A_{0}=0.0012 \mathrm{sq} . \text { miles }
$$

which is equivalent to a circular area of 210 feet diameter. The relative slip asso- 
ciated with an area of 210 feet diameter may be estimated as follows. The average slip varies with magnitude according to equation (2). For geometrically similar shocks the maximum relative slip is given by the same expression, that is,

$$
S=S_{0} c^{M}
$$

The maximum surface slip of the El Centro shock was approximately 15 feet, and if the maximum subsurface slip for a 6.7 magnitude, that is, the upper bound of a range of geometrically similar shocks, is taken to be the same, the slip for shocks of smaller magnitudes is given by

$$
S=15 e^{(M-6.7)}
$$

For $M=0$ this gives a maximum slip of 0.25 inch. This indicates that the typical smallest dislocation is one of area corresponding to approximately 210 feet in diameter with a maximum relative slip between faces of $0.25 \mathrm{inch}$.

If the preceding equations are applied to a shock of 8.2 magnitude, such as the San Francisco earthquake of 1906 , there is obtained a total area of slip equal to $300 \times 55$ miles. The surface slip of the 1906 shock disappeared into the ocean north of San Francisco, but it appears that the total slip area was of the order of magnitude of the above-mentioned figure.

The total movement in California can be estimated by means of the foregoing equations. If the mean annual frequency distribution of shocks in California is taken to be the same per unit area as for southern California, ${ }^{4}$ there is obtained

$$
f=0.00086 e^{2(8.7-M)} .
$$

The area of slip per shock according to the preceding calculation is:

$$
A=0.0012 e^{2 M} \text { sq. miles }
$$

The average relative slip over the area is approximated by one-half the maximum slip, or

$$
\bar{S}=\frac{1}{2}(0.25) e^{M} \text { inches }
$$

The total mean annual slipping is given by the integral of $(f A \bar{S})$, and if this is assumed to be distributed uniformly over faults 30 miles deep and 700 miles long there is obtained for the mean annual relative shearing motion of the east and west boundaries of the state

$$
\int_{0}^{8.7} \frac{(0.00086)(0.0012) \frac{1}{2}(0.25) e^{17.6} e^{M}}{(30)(700)} d M=2.2 \text { inches per year }
$$

This may be compared with the estimate of a mean annual relative motion of approximately 2 inches per year that is based on triangulation surveys.

\footnotetext{
${ }^{4}$ Gutenberg and Richter, Seismicity of the Earth.
} 
The average duration of the strong motion at the epicenter can be estimated from the motion of a point along the fault. Since such an element of material undergoes a displacement it must be subjected to a force that accelerates and decelerates. If similarity between earthcuakes is assumed, the force is similar for earthquakes of various magnitudes, differing only in duration. Thus the motion of the point can be written

$$
\begin{aligned}
\frac{d^{2} S}{d\left(t^{\prime} / k\right)^{2}} & =F\left(t^{\prime}\right) \quad\left(t^{\prime} / k=t, \quad 0<t^{\prime}<t_{1}{ }^{\prime}\right) \\
\therefore k^{2} S_{1} & =C \\
\left(\frac{t_{1}^{\prime}}{t_{1}}\right)^{2} S_{1} & =C \\
t_{1} & =C_{1} \sqrt{S}=C_{2} e^{M / 2}
\end{aligned}
$$

where $S_{1}$ is the maximum slip. The duration estimated from seismograms by Gutenberg and Richter ${ }^{5}$ is

$$
\log _{10} t_{0}=a+\frac{M}{4}
$$

or

$$
t_{0}=C e^{M / 1.93}
$$

The foregoing values of slip areas, and the like, are not exact, but are only approximations that are used to show that the formulas give reasonable values when applied to the limits of their ranges and that they are not inconsistent with observations.

\section{Accelerograms Derived from Stress Pulses}

When the stresses along a fault are released, they radiate stress pulses which after reflection and refraction are recorded on an accelerogram on the surface of the ground. It will be assumed that the pulses reaching the strong-motion accelerometer are similar to those sent out by the release of a single stress dislocation, or, more particularly, that each pulse records as one cycle of a sine wave. It is not essential that the pulse be exactly one cycle of a sine wave; this is only a computational convenience. However, it is essential that the pulse be double-looped, for it will be shown that it is not possible to form a typical accelerogram with single-looped pulses such as one-half cycle of a sine wave. It is also necessary that the swarm of pulses have various wave lengths, for it is not possible to form an accelerogram with the properties of recorded accelerograms with a swarm of pulses all of the same wave length. As will be seen, the pulses required are predominantly of $2 / 10$-second wave length.

It is assumed that during an earthquake the motion recorded by an accelerometer is composed of a swarm of pulses random in time. To determine the required distribution of pulse wave lengths and amplitudes the following method of analysis

\footnotetext{
${ }^{5}$ See reference in fn. 3 above.
} 
is used. A recorded accelerogram is considered to be a random continuous function and an earthquake is considered to be a random sample from a parent population. The characteristics of a random continuous function (accelerogram) are exhibited by its energy spectrum. The spectra have been computed for recorded strong-motion accelerograms and the average of these spectra is taken to be the spectrum of the parent population of strong-motion earthquakes. The distribution of pulse wave lengths, amplitudes, and numbers is determined so that the spectrum of the parent population of pulses is the same as the above-mentioned average recorded earthquake spectrum.

Consider a swarm of pulses, every one of which is represented by the acceleration

$$
f(k(t-\theta))=\sin k(t-\theta) \quad\left(\theta-\frac{\pi}{k}<t<\theta+\frac{\pi}{k}\right)
$$

where the times of occurrence $\theta$ are randomly distributed over an interval of time. If a certain number $n$ of such pulses are superimposed at random, they will form an accelerogram the energy spectrum of which is given by

$$
F(k, v, \theta)=\left(\int \sum f\left(k\left(t-\theta_{n}\right)\right) \sin v t d t\right)^{2}+\left(\int \sum f\left(k\left(t-\theta_{n}\right)\right) \cos v t d t\right)^{2}
$$

This will, in general, be an irregular curve, but the average of a large number of spectra from different sets of $n$ pulses will approach a smooth curve that characterizes the population from which the pulses were taken. To compute the average spectrum it is only necessary to integrate the foregoing expression with respect to $\theta$, since the probability of $\theta$ is constant with respect to time. When this is done, there is obtained for the average spectrum

$$
F(k, v)=2 \frac{n}{k^{2}}\left(\frac{\sin \pi \frac{v}{k}}{1-\left(\frac{v}{k}\right)^{2}}\right)^{2}
$$

or

$$
F(k, v)=2 \frac{n}{k^{2}}\left(\frac{\sin \frac{\pi}{r}}{1-\frac{1}{r^{2}}}\right)^{2}
$$

where $r$ is the ratio of the wave length being considered on the spectrum to the wave length of the pulses. This is shown in figure 3 , where the square root of the spectrum is drawn. In the case of one-half sine pulses, that is, single-loop pulses, the average spectrum is

$$
F(k, v)=2 \frac{n}{k^{2}}\left(\frac{\cos \frac{\pi}{2 r}}{1-\frac{1}{r^{2}}}\right)^{2}
$$

The square root of this curve is drawn also in figure 3 . These curves are typical in that the double-looped pulses produce a humped curve, whereas the single-loop pulses do not. 
If an accelerogram is formed by the random superposition of pulses of a variety of wave lengths and amplitudes, the mathematical expectation of the spectrum is

$$
F(k, v)=2 \sum_{k}\left(\frac{n_{k}^{1 / 2} A_{k}}{k} \frac{\sin \frac{\pi}{r}}{1-\frac{1}{r^{2}}}\right)^{2}
$$

where $n_{k}$ is the number of pulses in the population which have wave length $k$, and $A_{k}$ is the amplitude of these pulses.

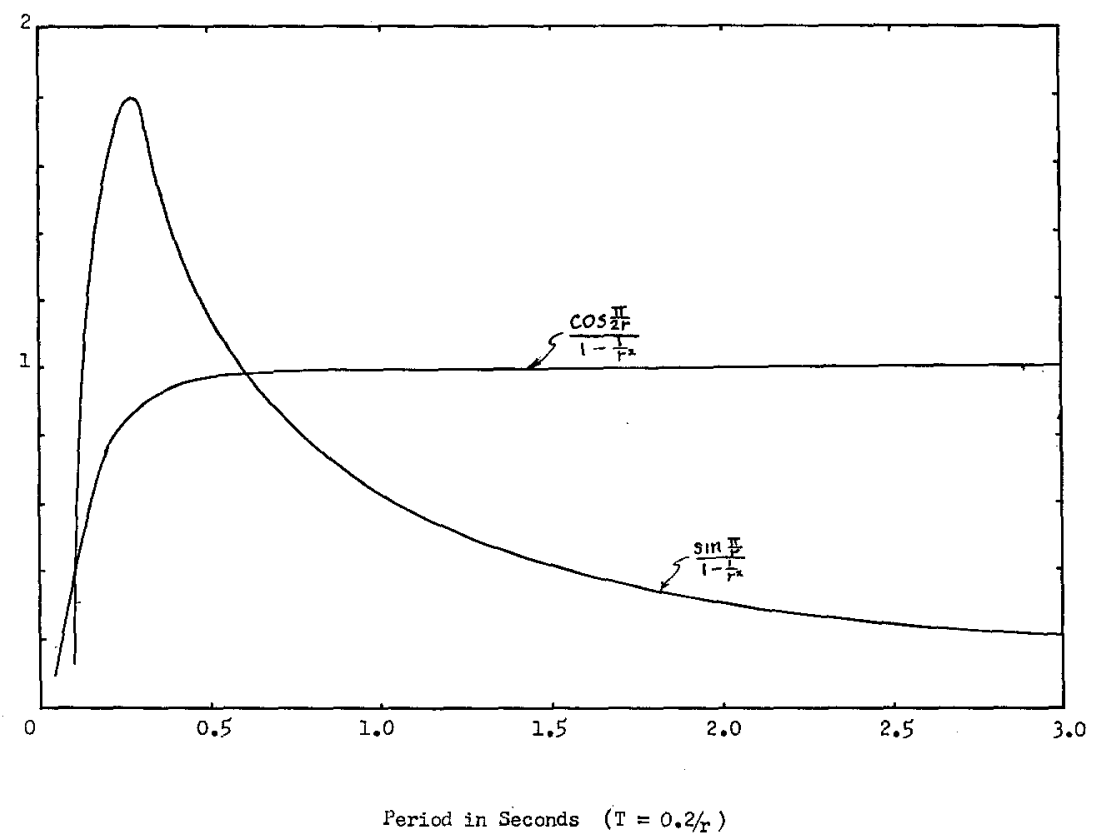

Fig. 3. Pulse spectra.

A typical earthquake spectrum is shown in figure 4. The spectrum shown is actually the square root of the energy spectrum. ${ }^{6}$ When the computed curves for the two components of the El Centro, May 18, 1940, El Centro, December 30, 1934, and Olympia, Washington, April 13, 1949, strong-motion accelerograms are averaged, there is obtained a fairly smooth curve that is closely approximated by the curve shown in figure 5. The averaging in this case was preceded by changing scales so that all of the individual spectra had the same average ordinate. The curve of figure 5 is taken to be the square root of the average energy spectrum. It is seen that the average spectrum has a hump and therefore cannot be derived from single-loop

${ }^{6}$ The random nature of earthquake ground motion was first pointed out in "Characteristics of Strong-Motion Earthquakes," Bull. Seism. Soc. Am., 37: 19-31 (1947). The spectra presented there were computed by means of a torsion pendulum that was later discovered to have approximately 0.01 of critical damping, which was sufficient to eliminate the hump in the average spectrum curve and reduce it to a horizontal line. Details of spectrum calculation will be found in J. L. Alford, G. W. Housner, and R. R. Martel, "Spectrum Analysis of Strong-Motion Earthquakes," Office of Naval Research Report', Contract N6onr-244, California Institute of Technology, August, 1951. 
pulses. It can, however, be derived from double-loop pulses. When equation (10) is fitted to the curve of figure 5 the values of $\left(n_{k}^{1 / 2} A_{k}\right)$ are determined, and these are shown in figure 6 . Since the numbers $n_{x}^{1 / 2}$ and the amplitudes $A_{k}$ occur as a product,

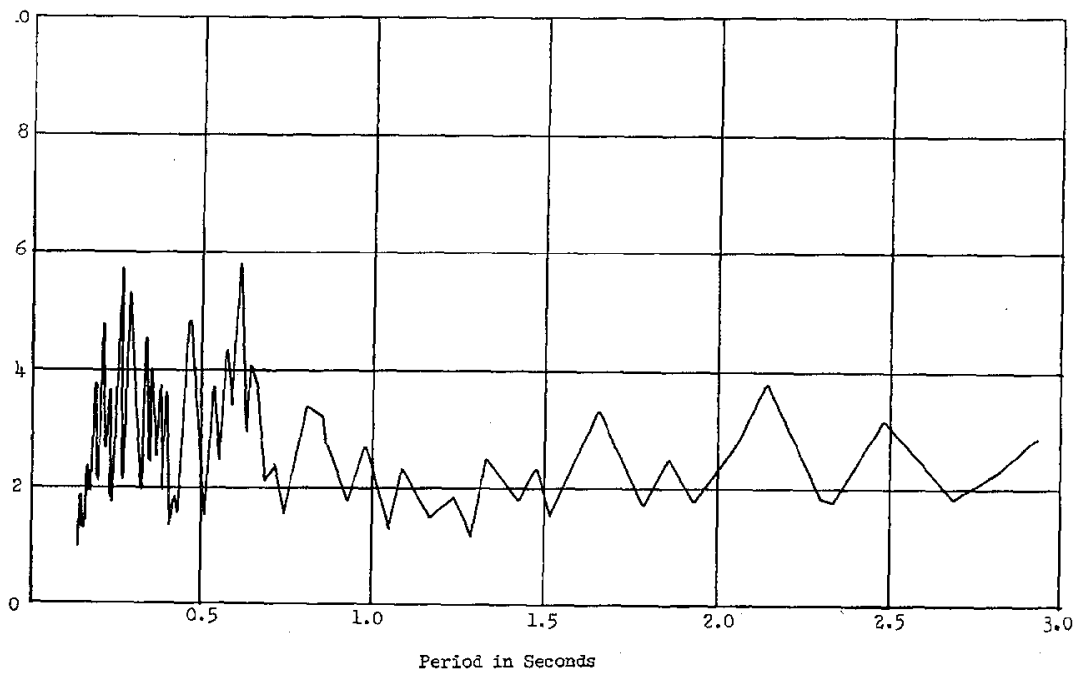

Fig. 4. Spectrum for Olympia, Wushington, earthquake of April 13, 1949. Component $\mathrm{S} 80^{\circ} \mathrm{W}$.

it is impossible to distinguish their separate effects on the spectrum, for quadrupling the number has the same effect as doubling the amplitude of the pulses.

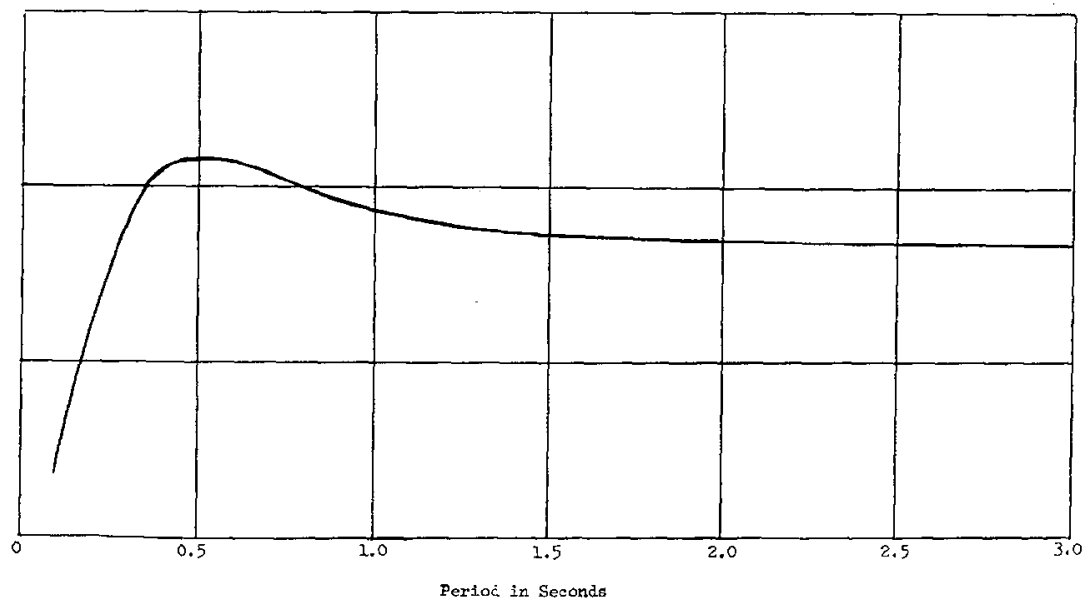

Fig. 5. Average spectrum for strong-motion earthquake.

The parent population of pulses may thus be considered to be composed of pulses of various wave lengths, having the amplitudes and numbers as given by figure 6 . If a random sample of $n$ of such pulses is taken and the sample is distributed at random over an interval of time, an accelerogram will be formed. The average 
spectrum of a large number of accelerograms obtained in this way will approach the spectrum of figure 5. A sample accelerogram obtained in this way will thus have the characteristics of actual recorded accelerograms so far as these are random continuous functions.

A sample accelerogram was constructed by distributing 584 pulses over a 10 second interval, allotting 292 to each 5 -second interval. To simplify the computa-

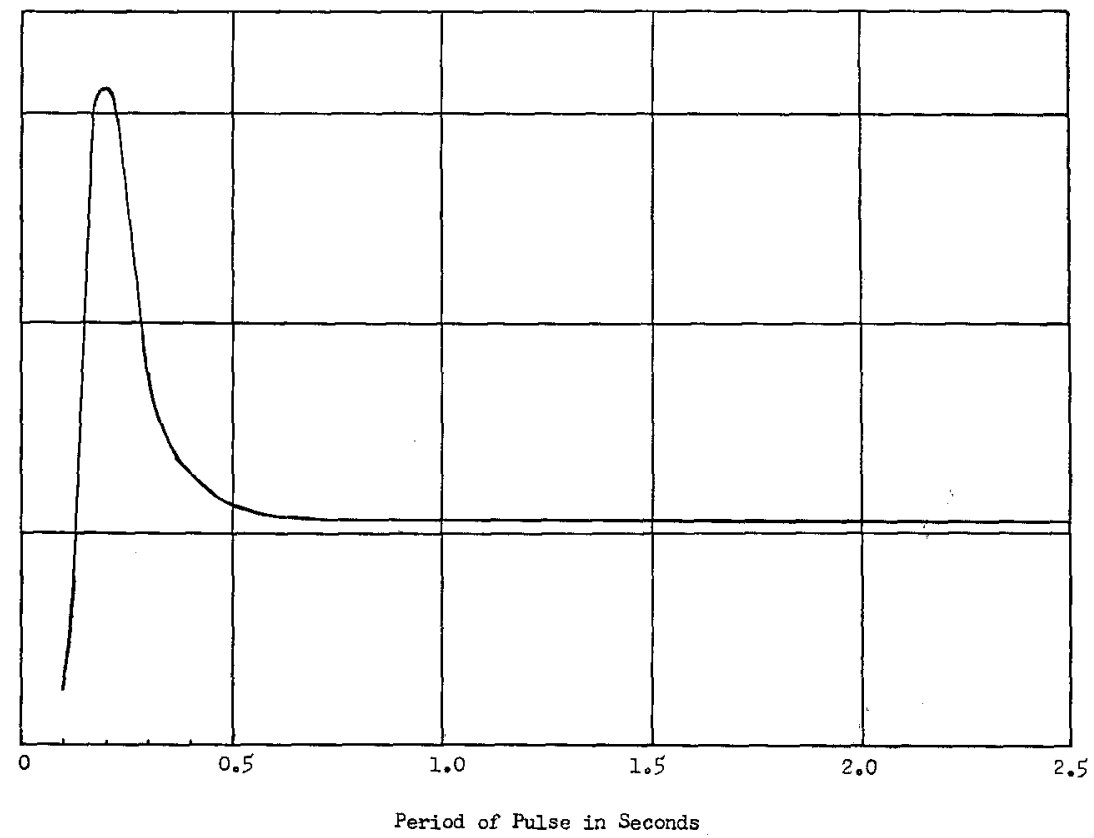

Fig. 6. Distribution of $n A^{2} / k$.

tions, the numbers and amplitudes of pulses for $1 / 10$-second increments of wave length were selected so that the proper $n_{k}^{1 / 2} A_{k} / k$ was obtained, and these were distributed at random by means of a table of random numbers. This procedure will give a somewhat more uniform-looking accelerogram than would be obtained if the pulses had been selected at random from the population and distributed at random over a 10-second period. The resulting accelerogram is shown in figure 7 . For comparison there is shown a portion of the Olympia, Washington, accelerogram in figure 8 . It is seen that the two are very similar in appearance even to the degree that on the average they cross the axis the same number of times per second.

It should be noted that the preceding method of superposing the pulses actually corresponds to the time of strong motion only, that is, when very large numbers of pulses are arriving. The earlier and later portions of recorded accelerograms may show many fewer pulses arriving. Also, one would infer that the random arrival of the longer-period pulses is a consequence of reflections and refractions through various strata; there is a similar effect on the short-period pulses. However, the character of the accelerogram depends largely on the predominant pulses having periods in the neighborhood of 0.2 second, and this is true for all the recorded strong-motion 


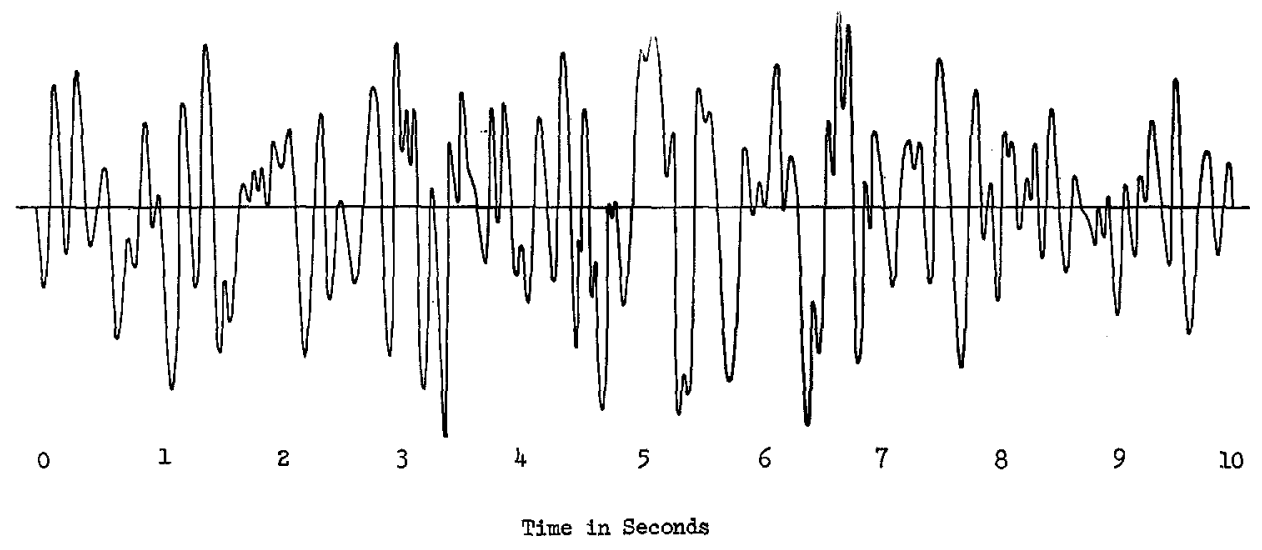

Fig. 7. Accelerogram formed by random superposition of pulses.

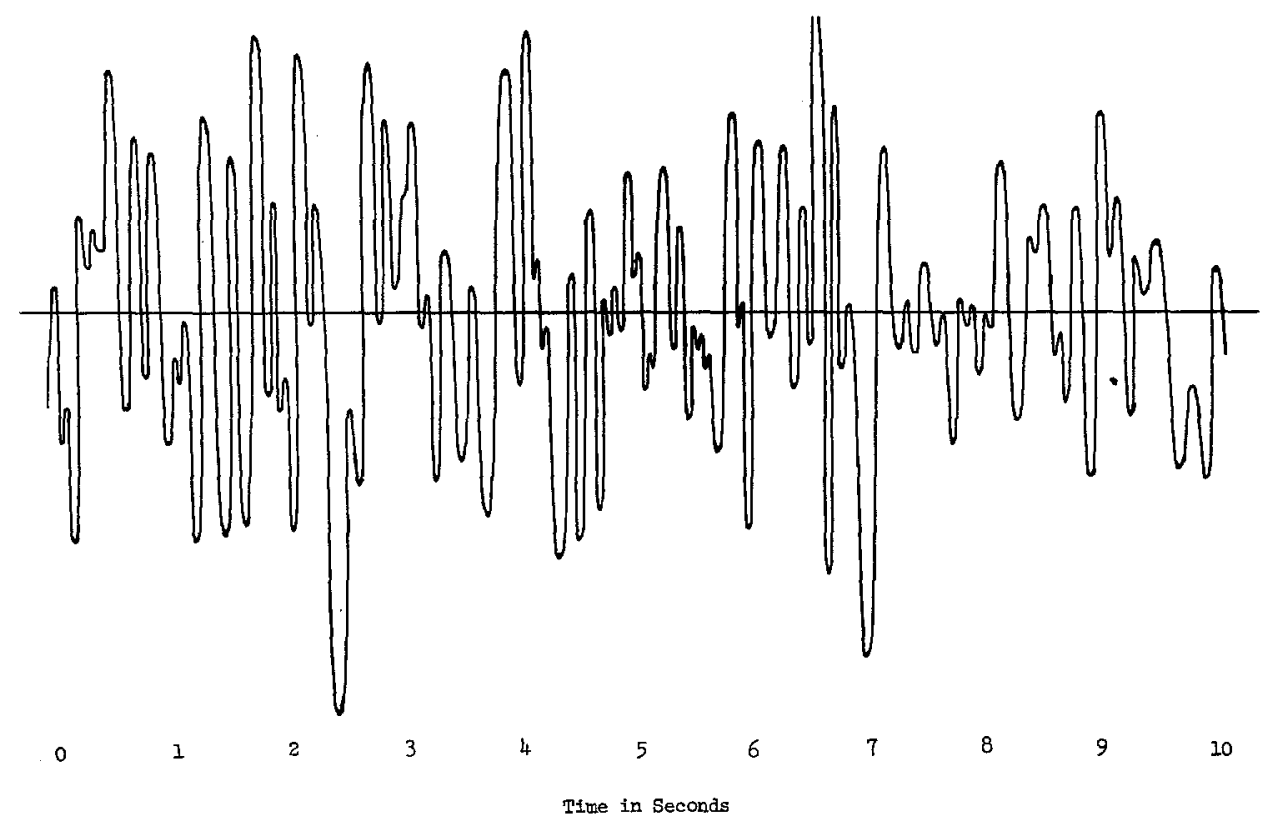

Fig. 8. Portion of Olympia, Washington, accelerogram.

accelerograms of large earthquakes, even though the shocks occurred at widely separated locations and had different types of slipping. It thus appears unlikely that this similarity can be attributed to reflections and refractions, but it may be a consequence of irregularities of stress release along the fault; for example, the original stress along the fault may be nonuniform or the stress may not be uniform during slipping, and the effects of these could be expected to be similar for different shocks. The effects of these short-period components would not, of course, be apparent on displacement records. 


\section{MaXimum ACCELERATIONS}

When an accelerogram is constructed with a set of pulses in the foregoing manner it is found that, to a considerable degree, the pulses cancel each other and that the maximum accelerations are the result of fortuitous superpositions of pulses. If a second accelerogram is constructed using the same set of pulses, it will differ from the first because of the random superposition of the pulses. However, if a large number of accelerograms are constructed using the same set of pulses, they will have certain average properties; for example, there will be an average maximum acceleration and the maximum accelerations of the individual accelerograms will deviate from the average with a certain statistical distribution. In what follows, the references to accelerations are to the average properties.

When the average energy spectrum is calculated for a given distribution of pulses, its ordinates, as shown by equation (10), are proportional to $\left(n A^{2}\right)$, where $n$ is the number of pulses and $A$ is the amplitude. On the other hand, if accelerograms are constructed from the pulses and the spectra computed for these, the average spectrum will have ordinates proportional to the square of the maximum acceleration. Therefore, the maximum acceleration $\alpha$ is proportional to the amplitude of the pulses and to the square root of the number of pulses, that is,

$$
\alpha=K(n)^{1 / 2} A
$$

where the number $n$ is the pulse density at the point where the accelerations are measured, that is, the number of pulses per unit time. If an increment of fault area $d x d h$ is considered to radiate pulses at a rate $n$ per unit area, the maximum acceleration at a specified point on the surface of the ground will have a maximum acceleration

$$
=K(n d x d h)^{1 / 2} A
$$

If the point on the surface of the ground is at such a distance from the fault that the radiation can be considered to come from a point source, the maximum acceleration recorded will be the cumulative acceleration from the total area that is radiating, that is,

$$
\alpha^{2}=K^{2} \iint n A^{2} d x d h
$$

If the radiation is the same from all points on the fault area, the maximum acceleration will be

$$
\alpha=C A(N)^{1 / 2}
$$

where $N$ is the rate of pulse radiation of the entire fault area and $A$ is the amplitude. $N$ is directly proportional to the area, so the maximum acceleration is proportional to the square root of the fault area. In terms of the magnitude

$$
\alpha=C_{1} e^{M}
$$

This is essentially Richter's definition of magnitude, in terms of acceleration instead of displacement, which is thus a measure of the area of slip. 
For points relatively close to the fault it is not correct to assume point-source radiation, but the area of the fault must be taken into account. A qualitative investigation of this effect may be made as follows. Consider a fault of length $2 l$ and vertical dimension $h_{0}$, each point of which is radiating pulses according to equation (11). Furthermore, let the effect of the position of the point on the surface of the ground, where the accelerations are measured, relative to the point on the fault be described by the inverse-square law with cosine correction as given by Gutenberg and Richter, ${ }^{7}$ that is,

$$
\alpha=K(n d x d h)^{1 / 2} A \frac{h}{x^{2}+y^{2}+h^{2}}
$$

where $x, y, h$ are the coördinates of the point on the surface of the ground with respect to the radiating point. The effect of the radiation from the fault area can be approximated by considering the radiation from a line of length $2 l$ at an equivalent depth $h$ if the strength of the radiation is taken to be proportional to $h_{0}$. If, then, the maximum acceleration is calculated for the line in accordance with equations (12) and (14), there is obtained

$$
\begin{aligned}
\alpha=\frac{\alpha_{0} h_{0}{ }^{1 / 2}}{2^{1 / 2}} \frac{h}{\left(h^{2}+y^{2}\right)^{3 / 4}}\left\{\frac{(x+l)\left(h^{2}+y^{2}\right)^{1 / 2}}{(x+l)^{2}+h^{2}+y^{2}}-\frac{(x-l)\left(h^{2}+y^{2}\right)^{1 / 2}}{(x-l)^{2}+h^{2}+y^{2}}\right. \\
\left.+\tan ^{-1} \frac{x+l}{\sqrt{h^{2}+y^{2}}}-\tan ^{-1} \frac{x-l}{\sqrt{h^{2}+y^{2}}}\right\}^{1 / 2}
\end{aligned}
$$

where $x, y, h$ are the coördinates of the point on the surface of the ground as measured in a coördinate system with origin at the center of the line, $x$ and $y$ being respectively parallel and perpendicular to the line and $h$ being the vertical distance from the center of the line to the surface of the ground. Equation (15) describes the variation of seismicity over the surface of the ground. When $l \rightarrow 0$ with $\left(\alpha_{0} l^{l / 2}\right)$ constant, there is obtained for point-source radiation

$$
\alpha=\alpha_{0}\left(h_{0} l\right)^{1 / 2} \frac{h}{\left(x^{2}+y^{2}+h^{2}\right)}
$$

which agrees with equation (14).

According to equation (13), $\alpha=C_{1} e^{M}$ and according to equation (4) the energy released is $E=C_{2} e^{3 M}$ so $\alpha$ is proportional to the cube root of the energy, and equation (16) can be written

$$
\alpha=C(E)^{1 / 3} \frac{h}{x^{2}+y^{2}+h^{2}} .
$$

The same equation is derived by Gutenberg and Richter ${ }^{8}$ by using empirical equations based on observations. For very large earthquakes equation (4a) shows $E=C_{2} e^{4 M}$, so the exponent in (16a) should be $1 / 4$ instead of $1 / 3$. This reflects the

\footnotetext{
${ }^{7}$ B. Gutenberg and C. F. Richter, "Earthquake Magnitude, Energy, Intensity and Acceleration," Bull. Seism. Soc. Am., 32: 163-191 (1942).

8 Ibid.
} 
fact that for very large shocks a significant part of the energy is released in longperiod waves which have very small accelerations.

According to equation (15), if $\frac{l}{\left(h^{2}+y^{2}\right)^{1 / 2}}$ is smaller than approximately 0.20 the fault may be considered to be a point source, but if the accelerations are measured closer to the fault than this the effect of the dimensions of the fault must be taken into account. For example, consider a point directly above the center of the fault at $x=0, y=0$. Equation (15) is then

$$
\alpha=\alpha_{0}\left(\frac{h_{0}}{h}\right)^{1 / 2}\left\{\frac{1}{\frac{h}{l}+\frac{l}{h}}+\tan ^{-1} \frac{l}{h}\right\}
$$

When $l / h$ is less than 0.2 equation (17) can be written approximately

$$
\alpha=\alpha_{0}\left(\frac{h_{0}}{h}\right)^{1 / 2}\left(\frac{l}{h}\right)^{1 / 2}
$$

and the radiation may be considered as originating from a point source. On the other hand, when $l / h$ becomes greater than this the effect of the increased length of fault upon the maximum accelerations rapidly decreases, as is shown in the table. It is seen that $\alpha$ calculated from equation (17) is virtually unaffected by increase in $l$ beyond $l / h=1.2$, and hence that an earthquake such as that of El Centro, 1940, for which $l$ was approximately 20 miles and the equivalent $h$ perhaps 10 miles, the maximum accelerations in the vicinity of the center of the fault would not have been materially increased for an $l$ of 40 or more miles. Thus, as regards the effect of area of slip, the maximum accelerations associated with the El Centro shock are close to the maximum possible.

\begin{tabular}{c|c}
\hline \hline$/ h$ & $\frac{a}{a_{0}}\left(\frac{2 h}{h_{0}}\right)^{\frac{1}{3}}$ \\
\hline 0 & 0 \\
2 & .62 \\
4 & .85 \\
6 & .99 \\
8 & 1.08 \\
1.0 & 1.13 \\
1.2 & 1.17 \\
$\infty$ & 1.25 \\
\hline
\end{tabular}

\section{SUMMARY}

The analysis given here considers that an earthquake fault is formed by the superposition of a large number of incremental shear dislocations the sudden release of which produces the earthquake. It is postulated that during an earthquake the incremental dislocations are released in such a way that the average slip is proportional to the square root of the area of slip, and that the probability of release of 
individual incremental dislocations is such that the probability of a total slip area $A$ is inversely proportional to $A$. With these two postulates a frequency distribution of earthquakes is derived that agrees with observed data; the Richter magnitude is shown to be essentially a logarithmic measure of the average slip on a fault; and an expression is derived for the energy released by an earthquake that agrees with that derived from consideration of the energy carried in a wave train. Expressions are derived also for the areas of slip during earthquakes, the maximum relative slip, and the average annual, over-all shearing distortion of the state of California and these are in satisfactory agreement with observed behavior. It is assumed that an accelerogram is formed by the superposition of a large number of elemental acceleration pulses random in time. It is shown that this agrees with recorded accelerograms, and an accelerogram composed in this fashion is shown to have the characteristics of actual recorded accelerograms. It is also shown that the maximum ground accelerations in the vicinity of the center of the fault, so far as they are dependent upon the size of the slip area, have essentially reached their upper limits for shocks with areas of slip approximately equal to that associated with the El Centro earthquake of 1940.

California Institute of Technology

Pasadena, California 